\title{
Cellular automaton modelling of surface discharge dynamics for EMC problems
}

\author{
A. S. Adalev ${ }^{1 a)}$, M. Hayakawa ${ }^{1}$, D. I. Iudin ${ }^{2}$, N. V. Korovkin ${ }^{3}$, \\ E. E. Selina ${ }^{3}$, and V. Yu. Traktengerts ${ }^{4}$ \\ ${ }^{1}$ The University of Electro-Communications, Tokyo, Japan \\ ${ }^{2}$ Radiophysical Research Institute, Nizhny Novgorod, Russia \\ ${ }^{3}$ Saint-Petersburg Polytechnical University, Saint-Petersburg, Russia \\ ${ }^{4}$ Institute of Applied Physics, Nizhny Novgorod, Russia \\ a)alexei@whistler.ee.uec.ac.jp
}

\begin{abstract}
Surface charge distribution on aircraft windshields and front head shields of missiles is highly inhomogeneous and it may cause creeping discharge appearance. The discharge produces strong electromagnetic emission, which get into the bodies through apertures. A fractal model based on a cellular automaton network reproducing the dynamics of surface electrical charge distribution is proposed. The model allows us to obtain both integrated characteristics of the discharge and its local properties. The latter is of extremely importance for EMC applications.
\end{abstract}

Keywords: surface discharge, creeping discharge, cellular automaton, dynamic model, fractal dimension

Classification: Electromagnetic theory

\section{References}

[1] M. S. Naidu and V. Kamaraju, High Voltage Engineering, McGraw-Hill, 1996.

[2] H. J. Wiesmann and L. Pietronero, "Properties of laplacian fractals for dielectric breakdown in 2 and 3 dimensions," in Fractals in Physics, ed. L. Pietronero and E. Tosatti, pp. 151-157, North-Holland, 1986.

[3] N. Femia, L. Niemeyer, and V. Tucci, "Fractal characteristics of electrical discharges: experiments and simulation," J. Phys. D: Appl. Phys., vol. 26, pp. 619-627, 1993.

[4] E. M. Bazelyan and Yu. P. Raizer, Spark discharge, CRC Press, 1998.

[5] M. Tanaka, Y. Murooka, and K. Hidaka, "Nanosecond surface discharge development using the computer simulation method," J. Appl. Phys., vol. 61, no. 9, pp. 4471-4478, 1987.

[6] Y. Murooka, T. Takada, and K. Hidaka, "Nanosecond surface discharge and charge density evaluation. Part I: review and experiments," IEEE Electr. Insul. Mag., vol. 17, no. 2, pp. 6-16, 2001.

[7] J. M. Meek and J. D. Craggs, Electrical Breakdown of Gases, Clarendon Press, 1953. 
[8] V. P. Belosheev, "Self-organization of structure of the leader discharge along water surface," Zh. Tekh. Fiziki, vol. 70, no. 7, pp. 109-114, 2000.

[9] A. Bunde and S. Havlin, Fractals and Disordered Systems, Springer, 1996.

[10] K. Falconer, Fractal Geometry - Mathematical Foundations and Applications, Wiley, 1990.

\section{Introduction}

The discharge over dielectric surface is widely used in various industrial applications, namely for the generation of directed X-ray beams, in pulse light sources, in laser pumping and so on. A negative effect of the surface discharge is also common knowledge. The electromagnetic radiation produced by the discharge affects adversely technical systems and biological objects, besides, it deteriorates the performance of electrical insulation, speeds up its aging and destruction [1].

As compared to the stochastic models based on the laplacian fractals $[2$, 3], which should be considered as models of the irreversible growth, we treat a surface discharge as a dynamic system of the charge distribution over the dielectric surface. The system is modeled by means of a 2-D network of cellular automata. Properly tuned, it permits us to take account of such parameters as electrode surface geometry, the shape of apertures and slots, dielectric characteristics and to determine discharge electromagnetic radiation characteristics which is essential for EMC problems.

\section{Modelling of a surface discharge by means of 2-D network of cellular automata}

A discharge area is presented in Fig. 1, where the positive electrode has a needle form. With increasing voltage across the gap a streamer corona initiates in the vicinity of the needle electrode. The streamer phase is rather short in the surface discharge development [4] and streamers cannot propagate without a leader, which starts from the electrode following the paths prepared by streamers. Actually a clear streamer corona may be observed for the first 10-200 ns $[5,6]$. The time resolution for the further discharge development (leader phase) is microseconds.

The experimental results $[6,7]$ show that during the charge distribution process, an arborescent structure of the current threads appears. The structure disintegrates after charging the accessible dielectric surface. Then a new arborescent structure grows from the root of the tree (electrode 1) along the uncharged surface. Each of these specific structures is scaleable and self-similar, i.e. reveals the fractal properties $[8,9,10]$.

In the present work the dynamic system of the current threads is considered to be a dynamic transport system or a resource allocation system, which is rather common in occurrence $[9,10]$. In addition, the resource allocation is performed in an extremely optimal way if the system is in the state of self-organized criticality, i.e. allocating the resource, it generates structure 


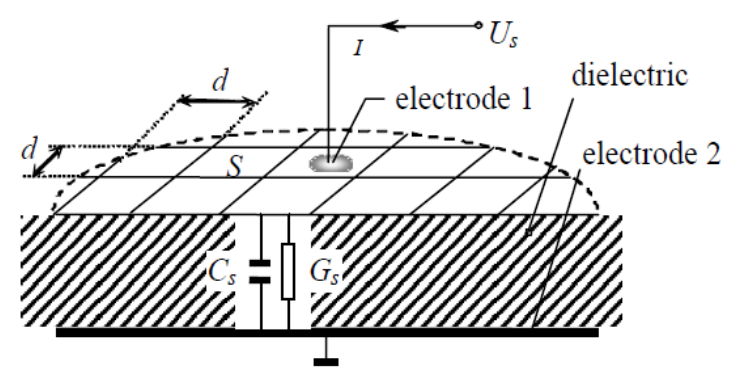

Fig. 1. Discharge Area.

elements of all possible scales. Low-scale structure elements appear and disappear relatively frequently but the life time of the large-scale elements is high and grows with their scale exponentially.

The modeling of the surface discharge is performed in the work by means of a 2-D cellular automaton network representing experimentally observed discharge characteristics. Each cellular automaton is associated with some dielectric surface element with area $d \times d$ (Fig. 1) and can assume one of the following three states: $\langle\mathrm{A} »,\langle\mathrm{~B} \Downarrow$ or $\langle\mathrm{C}\rangle$ which characterize the following automaton conditions:

A. Waiting condition. The capacitance $C_{S}$ of the element $S$ is not charged. All automata at the initial time instant are in the state $《 \mathrm{~A} \| \mathrm{ex}-$ cept the «zero» one (electrode 1 in Fig. 1 ) that is always in the state $《 \mathrm{C} »$.

B. Relaxation condition. The capacitor $C_{S}$ which was charged before to the voltage $U_{S}$, discharges exponentially in time. Automata in state B simulate positive ions left by the discharge.

C. Conducting condition. The automata in this state simulate the conductive streamer (leader) channel surrounded by positive ions. Following this channel, electrons produced at the streamer tip move toward the needle electrode [7].

An automaton can transit from the state $« \mathrm{~A} »$ only into the state $\langle\mathrm{B} »$. This occurs if one of his neighbors is in the state $\langle\mathrm{C}\rangle$ and the neighbor has excessive charge $Q>q_{0}$, where $q_{0}=C_{S} U_{S}$, where $U_{S}$ is an initiation threshold. As a result of the transition, the charge $q_{0}$ is transferred to the newly created automaton $« \mathrm{~B} »$.

An operational algorithm in the state «B» provides the discharge of the cell capacitor $C_{S}$ across the conductivity $G_{S}$ (Fig. 1). The latter, which is mostly connected with different types of particles recombination in plasma, is characterized by the time constant $\tau_{d i s}=C_{s} / G_{s}$. The well known first order finite-difference approximation of the capacitor discharge differential equation is used for simulation. In steady-state conditions the charge and discharge processes are in dynamic equilibrium.

The automaton transits from the state $\langle\mathrm{B} \|$ to the state $\langle\mathrm{A} \|$ on reaching some voltage threshold $U_{\text {min }}$. Being asked, a $\left.《 \mathrm{~B}\right\rangle$-state automaton generates the request for charge $\Delta q_{S}=q_{0}-q_{S}^{(N)}$, where $q_{S}^{(N)}$ is an instantaneous charge of the cell. This request is satisfied only if one of his neighbors is in the state $« \mathrm{C} »$ with sufficient extra charge $Q>q_{0}$. Otherwise, the request is canceled. 
For several recipients, the problem of charge distribution is solved randomly taking into account that the priority of $\langle\mathrm{B} \|$-automata is higher than that of 《A $》$-ones.

At the first time step, the «zero》 automaton receives the charge $m q_{0}$ from the external source. This charge is distributed randomly among the neighboring automata that are in the state $\langle\mathrm{A}\rangle$ (so they transit to the state $\langle\mathrm{B} »)$. Then it is verified that all the charge obtained from the source is distributed. If this is true, the time step ends. Otherwise, the remaining charge is distributed additionally. During this procedure some neighboring $\langle\mathrm{B}\rangle$-state automata are transferred into the state $\langle\mathrm{C}\rangle$ to deliver the remaining charge farther to their neighbors. The latter in their turn change the state from $\langle\mathrm{A} \Downarrow$ to $\langle\mathrm{B}\rangle$ receiving the charge. Following this way, the $\langle\mathrm{C}\rangle$-state automata form the arborescent cluster (system of streamers) distributing the charge over the dielectric surface.

The allocation process for the charge $m q_{0}$ is carried out until the remainder becomes less than $q_{0}$ or ceases to vary. The latter corresponds to the case when the given discharge tree exhausts its charge distributing capacity. The charge transferred by a $\langle\mathrm{C}\rangle$-state automaton is calculated at the end of each time step. If this value exceeds a certain threshold $q_{\text {pass }}$, the automaton remains in the state $\langle\mathrm{C}\rangle$, otherwise it is transferred into the state $\langle\mathrm{B} \|$. So, if a tree has exhausted its allocation capacity, it will be destroyed at the next time step, as no charge will be transferred through its branches.

Considering the charge transfer by $\| \mathrm{C}\rangle$-state automata as a current, the factor of loss in the discharge channel is also included into the model as follows. Let us consider that each $\langle\mathbf{C}\rangle$-state automaton in a cluster takes a piece of charge during charge transfer through it. The total amount of cells in the cluster may be estimated as $(R / d)^{D_{f}}$, where $R$ is a cluster geometric dimension and $D_{f}$ is the fractal dimension of the cluster, which may be easily determined. Therefore one may introduce a total charge loss at the $N$-th time step as $q_{\text {loss }} \sim k_{N} q_{0}(R / d)^{D_{f}}$, where the proportionality constant is a model parameter.

\section{Parameters and properties of the cellular automaton model}

The developed model involves a number of parameters whose determination is rather a complicate problem to be solved. By convention, the model parameters may be divided into the following three groups.

Physical parameters of the problem include those determined by electrical properties of the dielectric, electrode geometry and external conditions for the discharge existence. These parameters can be determined in fairly uncomplicated experiments or calculated with the sufficient accuracy.

Parameters determining source properties. Discharge evolution and dynamic equilibrium are substantially dependent on the type of the source delivering the charge to the «zero»automaton. In the present work all calculations were carried out under the assumption that the source is close to a voltage source. Consideration of a non-ideal source or charged capacitor 


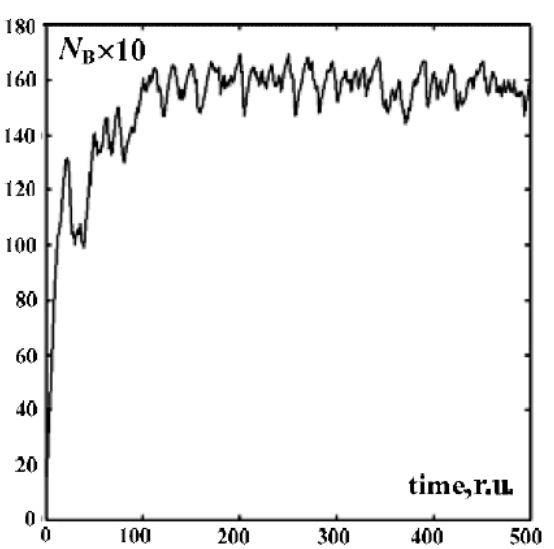

(a)

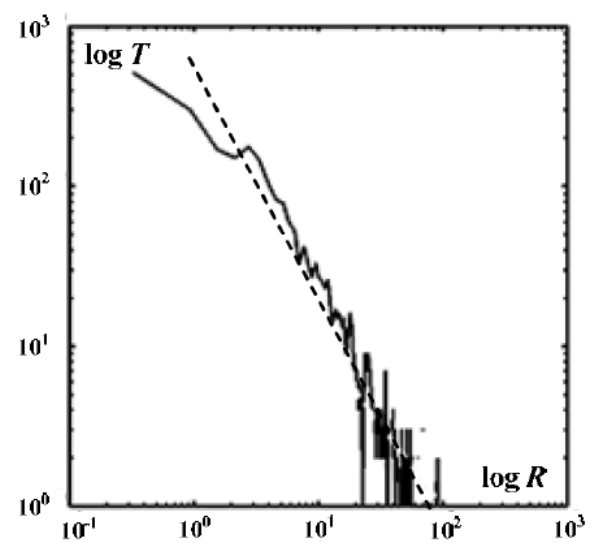

(b)

Fig. 2. Dynamic characteristics of the model: number of 《B$»$-cells versus time (a) and the total number of generated subclusters versus their size (b).

presents no substantial problems.

Parameters determining geometric characteristics of the cluster. These parameters determine a probability of discharge channel branching, a dominant direction of channel evolution and so on. Since the configuration of a cluster is closely related to the characteristics of the electromagnetic interference produced by the discharge, the parameters of this group may be properly adjusted by fitting the experimental characteristics of the discharge electromagnetic noise. The time scale of the model may be established in a similar way. The experimental dependence of the discharge current may be used to this end.

As noted above, surface charging and charge dispersion are in a dynamic equilibrium in the real surface discharge process (for a relatively long supplying time). In the proposed model these processes also come into equilibrium after certain initial time interval $\left[0, t_{\infty}\right]$. Fig. 2 (a) shows the time dependence of the number $N_{\mathrm{B}}$ of automata in the state $\langle\mathrm{B}\rangle$. Despite the substantial variation in the charge distribution structure (the shape of clusters consisting of the $« \mathrm{C} \Downarrow$-state automata) over the whole time period, the number of automata in the state $\langle\mathrm{B} »$ is relatively stable.

A histogram of the number $T$ of disintegrated and newly formed subclusters versus their size $R$ is given in Fig. 2 (b) in the bilogarithmic scale. The size of a subcluster was determined as a span between its most distant points. The histogram presented in Fig. 3 shows that during the simulation, clusters entire the whole range $\left[R_{\min }, R_{\max }\right]$ were produced. Here the simulation was made on a 2 -D mesh of $200 \times 200=4 \cdot 10^{4}$ cellular automata with cell size $d$ was assumed to be unity. Under these conditions $R_{\max }=100 \sqrt{2} \cong 142$, $R_{\min }=0.5$. Note that the dependence $\log T=f(\log R)$ is close to a linear one with a negative slope. This allows us to assume that the system is close to the state of self-organized criticality when the charge distribution is performed in the most effective way [10].

Typical pictures of simulated surface discharge patterns are shown in 

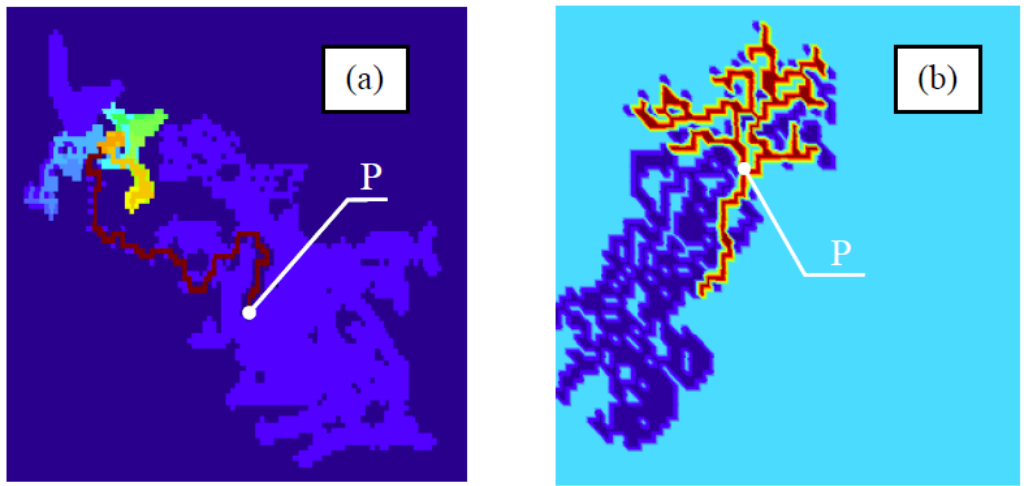

Fig. 3. Typical simulated patterns: single (a) and arborescent cluster (b).

Fig. 3. Point $\langle\mathrm{P}\rangle$ corresponds to the location of the «zero» automaton. The cluster (conductive channel) is shown in red. The scale of Fig. 3 (b) is twice smaller than that of Fig. 3 (a).

The model parameters for the process presented in Fig. 3 (a) are chosen in such a way that the channel branching is low and the losses are insignificant. Under these circumstances the existence of several channels is unlikely and the only one channel may be very long.

Similar pictures of surface discharge are observed under increased gas pressure [8]. Dark colors correspond to the automata in the state «A», lighter ones up to yellow depict the automata in the state $\langle\mathrm{B} »$. The lighter the field is, the larger is the remaining automaton charge. The cells of the cluster near the needle electrode are moving slowly in contrast to the fast moving cluster tail.

An instant picture of the branching channel is presented in Fig. 3(b). Here the light background corresponds to the uncharged cells, the dark one refers to the charged and discharging ones. Such a branched discharge cluster is observed for the near-normal and low gas pressures [7].

Average values of the discharge current and the number of the $《 \mathrm{C}\rangle$-state automata in the cluster are very close for the both cases. However, cluster mobility in the vicinity of the point $\langle\mathrm{P} »$ for the branched cluster (Fig. $3(\mathrm{~b})$ ) is considerably higher than that for the one presented in Fig. 3 (a). Accordingly, the radiation and current spectrums for the case of Fig. 3(b) are shifted towards higher frequencies.

\section{Conclusion}

The model proposed in the paper reproduces local characteristics of the surface discharge. An important feature of the model for the application to EMC problems is the possibility of obtaining the discharge current distribution over the dielectric surface. This allows us to determine characteristics of electromagnetic radiation in apertures and slots. Moreover, properly tuned, the model may also easily take into consideration geometric features of electrodes and dielectric, so that we can examine their effect on the discharge radiation. 
The created model is substantially dependent on the variety of model parameters whose adequate determination is a subject for the future research which involves experimental investigations. 